\title{
Frustrations and phase transitions in magnets of various dimensionality
}

\author{
Felix Kassan-Ogly ${ }^{1, \star}$ and Alexey Proshkin ${ }^{1}$ \\ ${ }^{1}$ Institute of Metal Physics of Ural Branch of Russian Academy of Sciences, 620990, Ekaterinburg, Russia
}

\begin{abstract}
We studied magnetic orderings, phase transitions, and frustrations in the Ising, 3-state Potts and standard 4-state Potts models on 1D, 2D, and 3D lattices: linear chain, square, triangular, kagome, honeycomb, and body-centered cubic. The main challenge was to find out the causes of frustrations phenomena and those features that distinguish frustrated system from not frustrated ones. The spins may interrelate with one another via the nearest-neighbor, the next-nearest-neighbor or higher-neighbor exchange interactions and via an external magnetic field that may be either competing or not. For problem solving we mainly calculated the entropy and specific heat using the rigorous analytical solutions for Kramers-Wannier transfer-matrix and exploiting computer simulation, par excellence, by Wang-Landau algorithm. Whether a system is ordered or frustrated is depend on the signs and values of exchange interactions. An external magnetic field may both favor the ordering of a system and create frustrations. With the help of calculations of the entropy, the specific heat and magnetic parameters, we obtained the points and ranges of frustrations, the frustration fields and the phase transition points. The results obtained also show that the same exchange interactions my either be competing or noncompeting which depends on the specific model and the lattice topology.
\end{abstract}

\section{Methods and basic formulae}

All considered models on all the lattices are described by the Hamiltonian:

$$
\mathcal{H}=-\frac{J}{2} \sum_{i, \Delta}\left(\mathbf{s}_{i} \cdot \mathbf{s}_{i+\Delta}\right)-\frac{J^{\prime}}{2} \sum_{i, \Delta^{\prime}}\left(\mathbf{s}_{i} \cdot \mathbf{s}_{i+\Delta^{\prime}}\right)-\sum_{i}\left(\mathbf{h} \cdot \mathbf{s}_{i}\right),
$$

where $J$ and $J^{\prime}$ mean exchange interactions between nearest-neighbor and next-nearest-neighbor spins; $h$ is an external magnetic field; sums run over lattice sites $i$, nearest-neighbors $\Delta$, next-nearest-neighbors $\Delta^{\prime}$, vectors $\mathbf{s}$ (with a module equal to unity) take on two, three, or four values, admissible in the Ising, 3-state Potts and 4-state Potts models.

If the analytical solution for maximum eigenvalue $\lambda$ of Kramers-Wannier transfer-matrix was found, the entropy $S$, specific heat $C$, magnetization $M$, etc. are only expressed in terms of $\lambda$ :

$$
S=\ln (\lambda)+\frac{T}{\lambda} \frac{\partial \lambda}{\partial T}, C=T \frac{\partial S}{\partial T}, M=\frac{T}{\lambda} \frac{\partial \lambda}{\partial h},
$$

In the absence of analytical solution, we performed the computer simulations by Monte-Carlo method, mainly in Wang-Landau algorithm [1].

\section{Types of frustrations}

Figures 1, 2 and 3 display several types of frustrations. The majority of papers in the $2 \mathrm{D}$ case are devoted to the

\footnotetext{
^e-mail: felix.kassan-ogly @imp.uran.ru
}

Ising model with competing antiferromagnetic nearest and next-nearest interactions on a square lattice (see, for example, [2]), frustrations are found at one point $J^{\prime} / J=0.5$. The phase transition point and the zero-temperature entropy $S(T=0)$ are equal to zero at this frustration point.

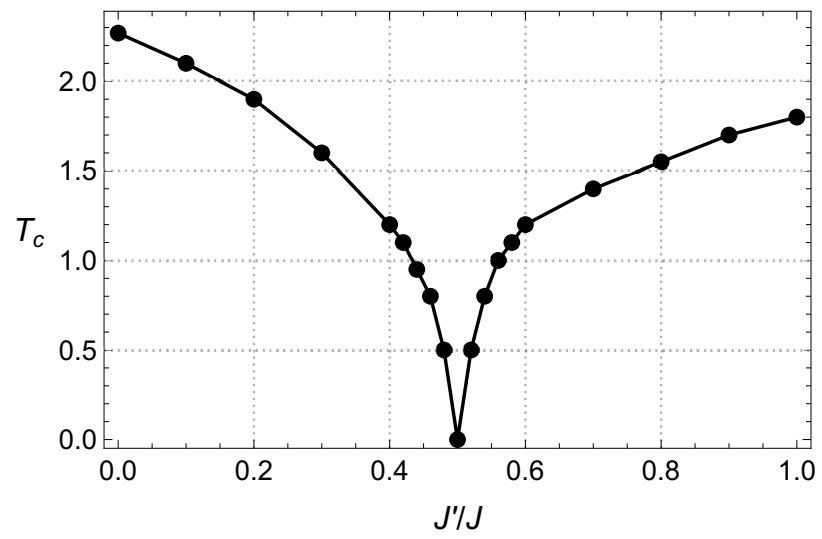

Figure 1. Phase transition temperature as a function of $J^{\prime} / J$ in Ising model on square lattice.

We studied Ising model with the same papameters on a body-centered cubic lattice [3] and obtain the phase diagram, shown in Fig. 2. The main difference of it from Fig. 1 is that the phase transition point has nonzero value, and this is another type of frustrations. We have discovered a novel (third) type of frustrations [4], shown in Fig. 3. First of all, in this type, a whole range of frustrations rather than a single frustration point is present. Second, despite the frustrations, the phase-transition temperature 


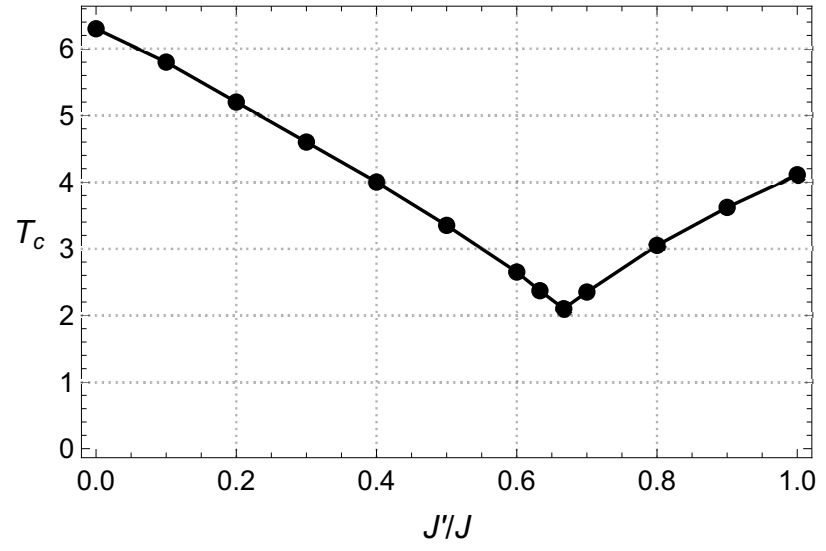

Figure 2. Phase transition temperature as a function of $J^{\prime} / J$ in Ising model on bcc lattice.

does not vanish at all. Third, the zero-temperature entropy $S(T=0)$ is nonzero and has one and the same value over the whole frustration range.

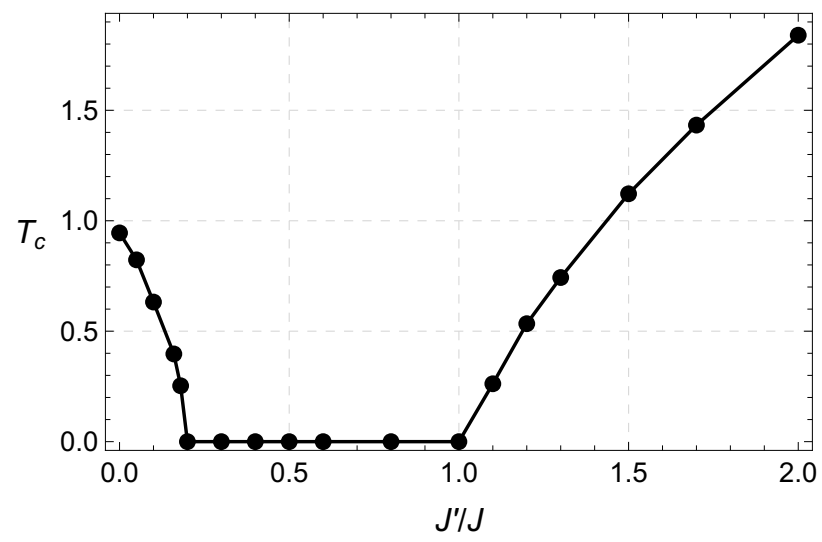

Figure 3. Phase transition temperature as a function of $J^{\prime} / J$ in 3 -state Potts model on a triangular lattice [4].

\section{Splitting of specific heat near frustration points}

We have revealed that frustrations create a remarkable peculiarity inherent to any model and any lattice. In the vicinity of every point of frustration or frustration field, specific heat splits into a sharp peak and a broad domeshaped peak. The phase-transition temperature decreases with approaching the frustration point or field. It may vanish, and the sharp peak disappears in this case at the frustration point or field. Otherwise, the broad dome-shaped peak disappears.

Using Onsager solution [5], keeping interaction along one side of a square $J_{x}$ constant and varying $J_{y}$ from -0.1 to +0.1 , we will obtain the temperature of phase transition as a function of $J_{y} / J_{x}$ (solid line in Fig. 4a), and the dependence of a broad peak maximum on $J_{y} / J_{x}$ (circles in Fig. 4a). Figure $4 \mathrm{~b}$ also shows specific heat at $J_{y} / J_{x}=0.01$.
The same behavior of heat capacity splitting takes place in all considered models on all the lattices considered, in particular, linear chain, triangular [6], and kagome lattice [7]. A very similar temperature dependence of magnetic contribution into heat capacity is observed in several real 3D crystals. Figure 5 displays, as an example, such heat capacity splitting in ErBi crystal [8]. As is seen, the authors' calculation attempt did not give an adequate agreement with experiment, since they were not aware that the effect has been created by frustrations.

The splitting of specific heat and decreasing of the phase transition temperature with increasing magnetic field towards frustration point were also observed in some real 3D crystals, for example, in TmTe [9] and in $\mathrm{Eu}_{0.95} \mathrm{Sr}_{0.05} \mathrm{Te}$ [10] crystals with magnetic face-centered cubic sublattices, but the frustration field had not been reached. Most probably, this was not done due to unawareness of the authors about such effect of frustrations.

\section{Partial ordering}

One more remarkable phenomenon, the so-called partial ordering (or ordering with lowered dimensionality) is observed in frustrated systems, which is closely related to the general properties of entropy. In the models considered, the entropy (per site) at $T \rightarrow 0$ equals to $\ln (n) / N$, where $N \rightarrow \infty$ is the number of lattice sites and $n$ is the number of configurations with the lowest energy, i.e. those that survive at $T \rightarrow 0$. If $n$ is finite, for example, in Ising model it is equal to 2 . Then entropy is equal to zero, and we have some kind of ordering. If $n$ is infinite and of the order of $q^{N}$ ( $q$ is the number of permissible states at a site) then the zero-temperature entropy is nonzero, and we have no ordering (frustrations). Sometimes $n$ can be computed, for example, in the standard antiferromagnetic 4-state Potts model on 1D chain it is equal to $4 \cdot 3^{N-1}$, and entropy is equal to $\ln (3)$.

The number $n$ may be infinite, but not of the order of $q^{N}$. The first example is the Onsager's case considered in the previous section. At $J_{y}=0$, and $J_{x}<0$ the system consists of separate chains, ordered antiferromagnetically and unlinked to one another. The number $n$ of such a system is equal to $2^{N_{y}}$, where $N_{y}$ is the number of sites along the $y$ direction in a square lattice. The zero-temperature entropy is equal to $N_{y} \ln (2) / N_{y} N_{x}$, or $\ln (2) / N_{x}$ and consequently, vanishes in the thermodynamic limit $\left(N_{x} \rightarrow \infty\right)$. As a whole, this 2D system has no finite transition point; nevertheless, in one direction it is ordered.

This phenomenon occurs very often in the systems with competing interactions, for example, in Ising model on a square lattice at the frustration-point value $J^{\prime} / J=0.5$, where $J$ is the antiferromagnetic exchange interaction between nearest-neighbor spins and $J^{\prime}$ is also the antiferromagnetic interaction between next-nearest-neighbor spins. It is more convenient to consider the pattern of partial ordering from the region, where $J^{\prime} / J>0.5$. Hereinafter, open and solid circles denote opposite spin directions. In this case, the magnetic structure looks like in Fig. 6a and is often called super-anti-ferromagnetic (SAF). It consists of alternating ferromagnetic columns in antiferromagnetic 


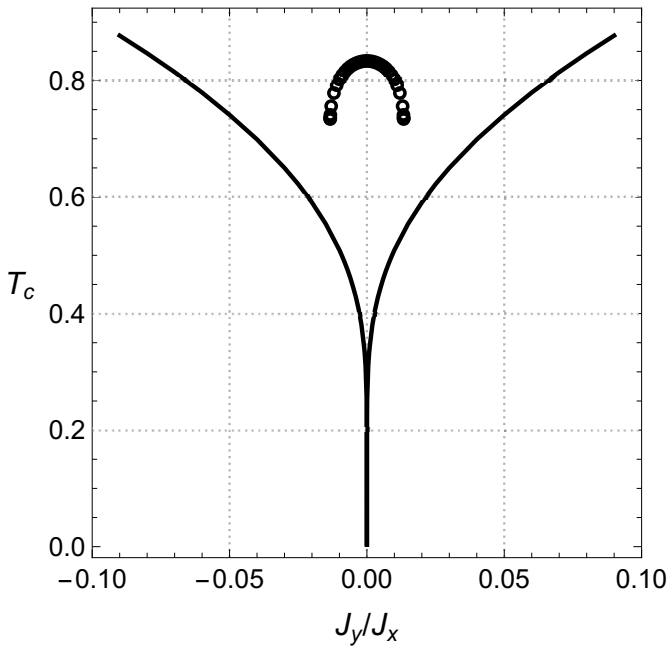

(a)

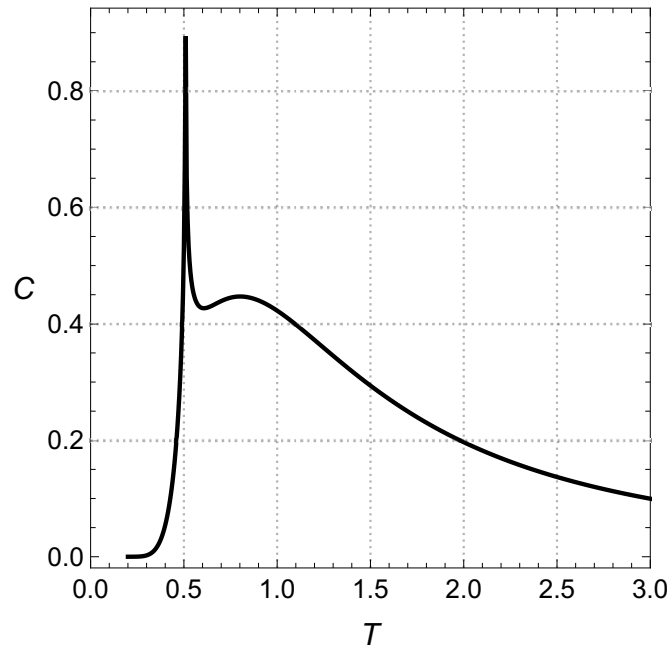

(b)

Figure 4. Phase transition temperature as a function of $J_{y} / J_{x}$ (a) and temperature dependence of heat capacity at $J_{y} / J_{x}=0.01$ (b) in Ising model on a square lattice.

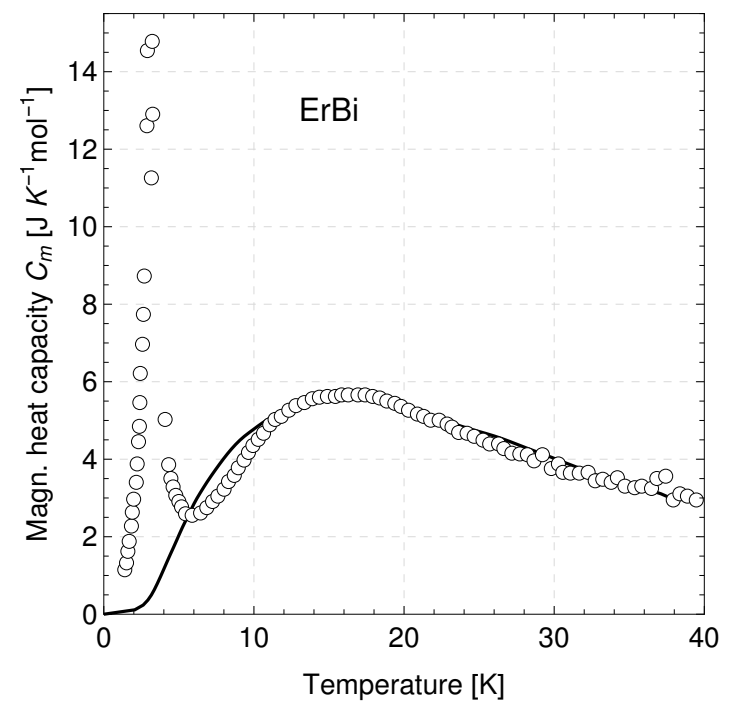

Figure 5. Magnetic heat capacity phase in ErBi. Circles - experiment. Solid line — calculation.

stacking. The partial ordering in this case is displayed in Fig. 6b. It consists of alternating ferromagnetic columns, but their stacking is violated. Calculating the internal energies of Néel structure, SAF structure, and the structure with stacking faults such as in Fig. 6b, we find out that all of them coincide.

In 3D systems, which consist of $N_{x} N_{y} N_{z}$ sites, the frustrations may create two possible kinds of partial ordering. The first is related to ordering in two dimensions that is the ordering within parallel planes and stacking faults of these planes. In this case, the zero-temperature entropy is equal to $\ln (q) / N_{z}$ and vanishes in the thermodynamic limit; the phase-transition temperature has non-zero value, and heat capacity has a form of sharp $\lambda$-shaped Onsager's peak. This is the partial ordering from $3 \mathrm{D}$ to $2 \mathrm{D}$ dimensionality.
The second kind of partial ordering corresponds to ordering in one dimension that is the ordering within parallel lines and stacking faults of these lines. In this case, the zero-temperature entropy is equal to $\ln (q) / N_{x} N_{y}$ and also vanishes in the thermodynamic limit. However, the phasetransition temperature vanishes, phase transition does not occur and heat capacity has gently sloping shape, as in all 1D lattices. This is the partial ordering from $3 \mathrm{D}$ to $1 \mathrm{D}$ dimensionality.

Our studies [3] in Ising model with competing antiferromagnetic $J$ and $J^{\prime}$ interactions on bcc lattice, in which the frustration point is equal to $J^{\prime} / J=2 / 3$, meets all the features of first-kind partial ordering. The $2 \mathrm{D}$ ordering takes place within planes perpendicular to cube spacediagonals of (111) type. The second kind of partial ordering is not yet found.

\section{Competing and noncompeting interactions}

It is commonly accepted that competing interactions may be of two kinds, either $J<0$ and $J^{\prime}<0$ or $J>0$ and $J^{\prime}<0$, and it is really valid for Ising model in all dimensions, for 3-state Potts model on a triangular lattice, and many others. However, $J<0$ and $J^{\prime}<0$ interactions appear to be favoring rather then competing, which strongly depends on the choice of a model and a lattice topology. The example of noncompeting $J<0$ and $J^{\prime}<0$ interactions in 4-state Potts model on a triangular lattice is shown in Fig. 7. Hereinafter, numbers in circles denote four permissible spin directions corresponding to the 4-state standard Potts model. They are such that any unlike pair makes one and the same angle. Both interactions demand nonparallel arrangement for all pairs of nearest and next-nearest spins, and these demands are simultaneously fulfilled in the Fig. 7, resulting in the absence of frustrations at any values of interactions. 


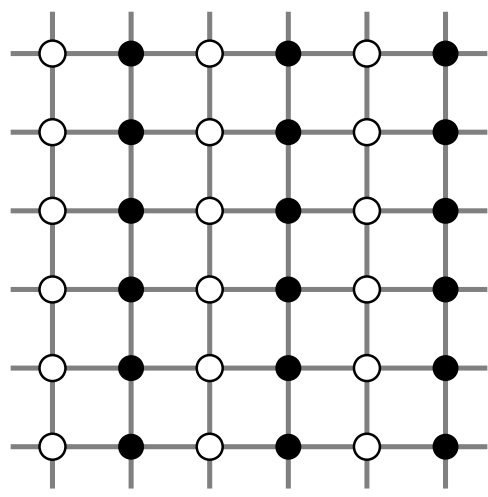

(a)

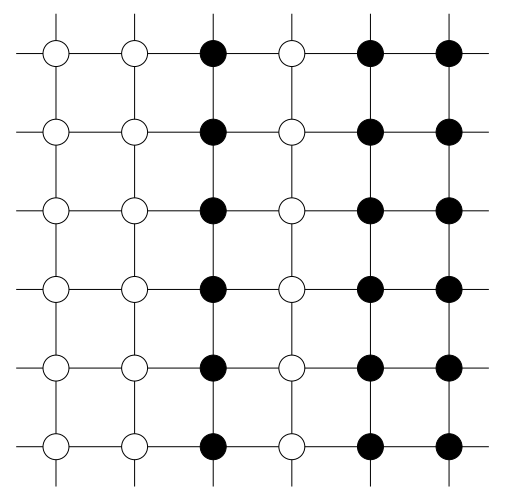

(b)

Figure 6. SAF ordering, $J^{\prime} / J>0.5$ (a) and partial ordering, $J^{\prime} / J=0.5$ (b) in Ising model on a square lattice.

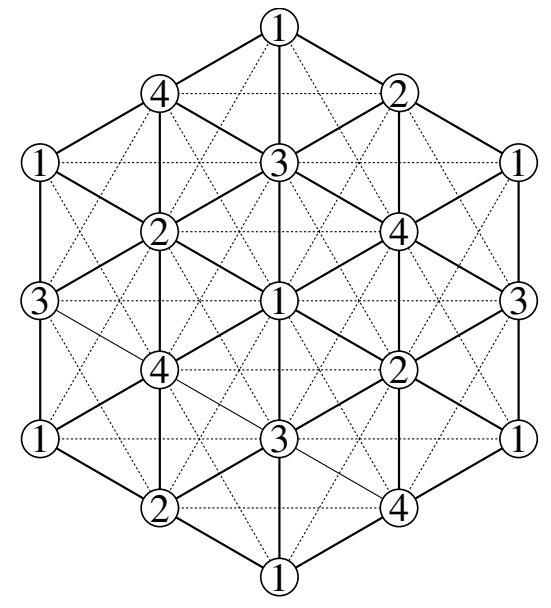

Figure 7. Noncompeting interactions on triangular lattice. 4-state Potts model.

\section{Summary}

We considered the Ising and Potts models with taking into account the nearest and next-nearest neighbor interactions on 1D, 2D, and 3D lattices: linear chain, square, triangular, kagome, honeycomb, and body-centered cubic. We have revealed many distinguishing features concerning magnetic orderings, phase transitions, and frustrations.

The competition between interactions and an external magnetic field may create either frustration points or phase transitions points, and sometimes even the ranges of frustrations. The interplay of competing interactions results sometime in the partial ordering (which means lowering of ordering dimensionality). This phenomenon occurs in Ising model on square, kagome, triangular, and bodycentered cubic lattice.

In some cases the same interactions may either be competing or noncompeting. For example, in 4-state Potts model, the antiferromagnetic nearest and next-nearest interactions on 1D chain compete so strong that lead to frustrations at any values, so that only the third-neighbor inter- action can create ordering. Quite the contrary, on a triangular or honeycomb lattice these interactions are noncompeting and lead to the phase transitions also at any values.

We predict that at appropriate choice of a model the magnetic properties of $1 \mathrm{D}, 2 \mathrm{D}$, and 3D lattices will be similar despite that linear chain does not possess a phase transition while $2 \mathrm{D}$ and $3 \mathrm{D}$ lattices do. The rescaling of the temperature and magnetic field is the necessary condition thereto.

The most astonishing result is that in every model on any lattice, in the vicinity of frustration point or frustration field the specific heat splits into a sharp peak and a broad dome-shaped peak, both of which move with the field variation.

The research was carried out within the state assignment of FASO of Russia (theme «Quantum» no. 01201463332).

\section{References}

[1] F. Wang, D.P. Landau, Phys. Rev. E 64, 056101 (2001)

[2] A. Kalz, A. Honecker, S. Fuchs, T. Pruschke, Eur. Phys. J. B 65, 533 (2008)

[3] A.K. Murtazaev, M.K. Ramazanov, F.A. KassanOgly, D.R. Kurbanova, JETP 120, 127 (2015)

[4] A.B. Babaev, M.A. Magomedov, A.K. Murtazaev, F.A. Kassan-Ogly, A.I. Proshkin, JETP 122, 310 (2016)

[5] L. Onsager, Phys. Rev. 65, 117 (1944)

[6] R.M.F. Houtappel, Physica 16, 425 (1950)

[7] K. Kanô, S. Naya, Prog. Theor. Phys. 10, 158 (1953)

[8] H. Wada, H. Imai, M. Shiga, J. All. Comp. 218, 73 (1995)

[9] T. Matsumara, H. Shida, T. Suzuki, Physica B, Condensed Matter 230-232, 738 (1997)

[10] E. Scheer, J. Wosnitza, H.v. Löhneysen, Z. Phys. B 85, 79 (1991) 